\title{
SYMPLECTIC AUTOMORPHISMS OF K3 SURFACES OF ARBITRARY FINITE ORDER
}

\author{
DANiEl Huybrechts
}

\begin{abstract}
It is observed that the existing results in [9] and [13] suffice to prove in complete generality that symplectic automorphisms of finite order of a K3 surface $X$ act as identity on the Chow group $\mathrm{CH}^{2}(X)$ of zero-cycles.
\end{abstract}

In [13], Claire Voisin recently proved that symplectic involutions of a K3 surface $X$ act trivially on the Chow group $\mathrm{CH}^{2}(X)$ of zero-dimensional cycles. We shall explain that this can be combined with a result in [9], which in turn is based on techniques involving the derived category of coherent sheaves $\mathrm{D}^{\mathrm{b}}(X)$ developed in [7], to prove the following:

Theorem 0.1. Let $f: X \stackrel{\sim}{\longrightarrow} X$ be a symplectic automorphism of finite order of a complex projective K3 surface $X$. Then $f^{*}=\mathrm{id}$ on $\mathrm{CH}^{2}(X)$.

As explained at various places before, the result can be seen as evidence for the much more general philosophy of Boch and Beilinson on filtrations of Chow groups and their relation to Hodge theory; see e.g., $[9,13]$.

The particular case of automorphisms $f: X \stackrel{\sim}{\rightarrow} X$ of a K3 surface with $f^{*}=$ id on $H^{2,0}(X)$ has been addressed explicitly in [7] in the context of autoequivalences of the derived category $\mathrm{D}^{\mathrm{b}}(\operatorname{Coh}(X))$ : if the induced action $f^{*}: H^{*}(X, \mathbb{Z}) \stackrel{\sim}{\longrightarrow} H^{*}(X, \mathbb{Z})$ (on the full cohomology!) of a symplectic automorphism $f: X \stackrel{\sim}{\rightarrow} X$ coincides with the action induced by a composition $\prod T_{E_{i}}$ of spherical twists

$$
T_{E_{i}}: \mathrm{D}^{\mathrm{b}}(\operatorname{Coh}(X)) \stackrel{\sim}{\rightarrow} \mathrm{D}^{\mathrm{b}}(\operatorname{Coh}(X)),
$$

it was shown that the action $f^{*}: \mathrm{CH}^{2}(X) \stackrel{\sim}{\longrightarrow} \mathrm{CH}^{2}(X)$ is trivial. Recall that the spherical twist $T_{E}$ associated with a spherical object $E \in \mathrm{D}^{\mathrm{b}}(X)$, i.e., an object with $\operatorname{Ext}^{*}(E, E) \cong H^{*}\left(S^{2}, \mathbb{C}\right)$, is the autoequivalence described by

$$
F \mapsto \operatorname{Cone}\left(\operatorname{Ext}^{*}(E, F) \otimes E \longrightarrow F\right) .
$$

The possibility of using this to prove $f^{*}=\mathrm{id}$ in general was studied in [9]. There, a condition on the 2-rank and the 3-rank of the Néron-Severi lattice $\operatorname{NS}(X) \subset H^{2}(X, \mathbb{Z})$ was formulated, that ensures that indeed for any symplectic automorphism $f^{*}=$ $\prod T_{E_{i}}$ on $H^{*}(X, \mathbb{Z})$ for some spherical twists. It was remarked that the condition on $\mathrm{NS}(X)$ is verified for a dense subset of $\mathrm{K} 3$ surfaces $(X, f)$ with a symplectic involution, but that it definitely fails in general. Since the case of symplectic involutions seemed the most approachable, this attack was abandoned at the time. Instead, we

Received by the editors July 6, 2012.

2000 Mathematics Subject Classification. xxx. 
tried in [12] to approach the case of involutions by more geometric arguments and succeeded in proving the theorem in one-third of all cases. The same techniques (existence of nodal elliptic curves via deformation theory of stable maps) apply more generally to symplectic automorphisms of higher order but yield the result only for some components of the moduli space of $(X, f)$.

Cases of symplectic involutions on K3 surfaces of low degree have also been studied in $[3,8,10,11]$, but the best result has been obtained by Voisin in [13]. Instead of working with invariant elliptic curves as in [12], she uses Prym varieties to deduce finite dimensionality (in the sense of Roitman) of the anti-invariant part of $\mathrm{CH}^{2}(X)$ which is enough to prove the result. Unfortunately, the same idea seems to fail for symplectic automorphisms of order $>2$, as the dimensions of the linear systems and the Prym varieties do not match up.

It is curious to note, that for automorphisms of prime order $p>2$, where the beautiful geometric arguments of [13] seemed to fail, the derived and less geometric techniques of [9] work without any further work (although they give only very partial results for involutions). In fact, the point of this note is to remark that the case of symplectic automorphisms of prime order $p>2$ could have been settled already in [9]. If only I had appreciated the very useful classification results of [5] more and had put them to better use. Eventually, combining the case $p=2$ with the cases $p>2$ yields the result for symplectic automorphisms of arbitrary finite order.

\section{Proof}

Let $X$ be a complex K3 surface and let $f: X \stackrel{\sim}{\rightarrow} X$ be a symplectic automorphism of prime order $p=\operatorname{ord}(f)$. Thus, the induced action of $f$ on $H^{2}(X, \mathbb{Z})$ is the identity on $H^{2,0}(X)$ and hence on the transcendental lattice $T(X)$. As was shown by Nikulin in [1] there are only the following possibilities: $p=2,3,5,7$. We will explain that Theorem 0.1 for symplectic automorphisms of prime order implies the results for all finite orders. In fact, only $n=2,3,4,5,6,7,8$ can occur.

1.1. If $H^{2}(X, \mathbb{Z})$ is considered as an abstract lattice, then the $f$-invariant part and its orthogonal complement have been studied in great detail by Nikulin [1], Morrison [2], van Geemen and Sarti [4], and Garbagnati and Sarti [5]. As it turns out, the invariant part $H^{2}(X, \mathbb{Z})^{f}$ and its orthogonal complement $\left(H^{2}(X, \mathbb{Z})^{f}\right)^{\perp}$ only depend on the order $p$ and can be described abstractly.

We shall briefly review the facts relevant for our purpose. The orthogonal complement of $H^{2}(X, \mathbb{Z})^{f}$ will simply be denoted

$$
\Omega_{p}:=\left(H^{2}(X, \mathbb{Z})^{f}\right)^{\perp} .
$$

Clearly, as for the symplectic automorphism $f$ the transcendental lattice $T(X)$ is contained in $H^{2}(X, \mathbb{Z})^{f}$, its orthogonal complement is contained in the Néron-Severi group:

$$
\Omega_{p} \subset \mathrm{NS}(X)
$$

Recall that $H^{2}(X, \mathbb{Z})$ is isomorphic to $U \oplus U \oplus U \oplus E_{8}(-1) \oplus E_{8}(-1)$.

(i) $p=2$. Then $H^{2}(X, \mathbb{Z})^{f} \cong U \oplus U \oplus U \oplus E_{8}(-2)$ and $\Omega_{2} \cong E_{8}(-2)$, which is a lattice of rank 8 and discriminant $2^{8}$, cf. [4, Sec. 1.3] or [5, Thm. 4.1]. 
(ii) $p=3$. Then $H^{2}(X, \mathbb{Z})^{f} \cong U \oplus U(3) \oplus U(3) \oplus A_{2} \oplus A_{2}$ and $\Omega_{3}$ is a lattice of rank 12 and discriminant $3^{6}$, cf. [5, Thm. 4.1, Prop. 4.2].

(iii) $p=5$. Then $H^{2}(X, \mathbb{Z})^{f} \cong U \oplus U(5) \oplus U(5)$ and $\Omega_{5}$ is a lattice of rank 16 and discriminant $5^{4}$, cf. [5, Theorem. 4.1, Prop. 4.4].

(iv) $p=7$. Then $H^{2}(X, \mathbb{Z})^{f} \cong U(7) \oplus\left(\begin{array}{ll}4 & 1 \\ 1 & 2\end{array}\right)$ and $\Omega_{7}$ is a lattice of rank 18 and discriminant $7^{3}$, cf. [5, Thm. 4.1, Prop. 4.6].

In [5] one also finds information e.g., on the discriminant form and the action of $f$ on it, but this is not needed here.

As usual, $U$ denotes the hyperbolic plane $\left(\begin{array}{ll}0 & 1 \\ 1 & 0\end{array}\right)$ and $E_{8}$ is the unique even unimodular positive lattice of rank 8 . For any lattice $\Lambda$, the twist $\Lambda(n)$ by an integer $n$ denotes the lattice that is given by $n(,)_{\Lambda}$, where $(,)_{\Lambda}$ is the pairing on $\Lambda$.

1.2. Recall that for a prime number $q$, the $q$-rank $\operatorname{rk}_{q}(\Lambda)$ of a lattice $\Lambda$ is the maximal rank of a sublattice $\Lambda^{\prime} \subset \Lambda$ whose discriminant is not divisible by $q$. For the application we have in mind, only the cases $q=2$ and $q=3$ will play a role.

As a consequence of the results of [5], one immediately finds the following

Corollary 1.1. (i) If $p=5$, then $\operatorname{rk}_{2}\left(\Omega_{5}\right)=\operatorname{rk}_{3}\left(\Omega_{5}\right)=16$.

(ii) If $p=7$, then $\operatorname{rk}_{2}\left(\Omega_{7}\right)=\operatorname{rk}_{3}\left(\Omega_{7}\right)=18$.

For $p=3$, the lattice $\Omega_{3}$ has maximal 2-rank, but its 3 -rank is strictly smaller than $\operatorname{rk}\left(\Omega_{3}\right)=12$, but the following weaker result will suffice.

Corollary 1.2. If $p=3$, then $\operatorname{rk}_{2}\left(\Omega_{3}\right)=12$ and $\operatorname{rk}_{3}\left(\Omega_{3}\right) \geq 3$.

Proof. The first assertion is obvious and the second follows from the explicit description of the intersection matrix of $\Omega_{3}$ given in [5, Prop. 4.2] in terms of a basis $e_{1}, \ldots, e_{12}$. We refrain from copying the matrix here, but a quick look shows that $e_{1}, e_{7}$ and $e_{11}$ (or, alternatively, $e_{1}, e_{5}$, and $e_{11}$ ) span a sublattice $\Lambda^{\prime}$ which modulo 3 has a diagonal intersection matrix $\operatorname{diag}(2,2,2)$, the discriminant of which is clearly not divisible by 3 .

Remark 1.3. For $p=2$, the 3 -rank of $\Omega_{2}$ is again maximal, but the 2-rank is zero. This explains why [9, Thm. 6.5] definitely does not apply to the generic symplectic involution.

1.3. For the convenience of the reader, we recall the following result, which is $[9$, Thm. 6.5].

Theorem 1.4. Suppose $\operatorname{rk}_{2}(\mathrm{NS}(X)) \geq 4$ and $\mathrm{rk}_{3}(\mathrm{NS}(X)) \geq 3$. Then any symplectomorphism $f \in \operatorname{Aut}(X)$ acts trivially on $\mathrm{CH}^{2}(X)$.

This theorem uses a result of Kneser, generalizing results of Wall for unimodular lattices, that shows that under the assumptions on $\mathrm{NS}(X)$ every orthogonal transformation of $\operatorname{NS}(X) \oplus H^{0}(X, \mathbb{Z}) \oplus H^{4}(X, \mathbb{Z})$ with trivial spinor norm and discriminant is a composition of reflections. But reflections can always be lifted to spherical twists. Note that the spherical twists $T_{E_{i}}$ that occur in the decomposition $f^{*}=\prod T_{E_{i}}^{*}$ will typically not respect $H^{2}$.

By (1.1) we know that $\mathrm{NS}(X)$ contains $\Omega_{p}$. Combined with Corollary 1.1, Corollary 1.2, and Theorem 1.4, this yields the main result of this paper: 
Corollary 1.5. Let $f: X \stackrel{\sim}{\longrightarrow} X$ be a symplectic automorphism of a projective $K 3$ surface $X$ and suppose that the order of $f$ is $p=3,5$, or 7 . Then the induced action of $f$ on $\mathrm{CH}^{2}(X)$ is trivial.

In order to conclude the proof of Theorem 0.1, one first invokes Nikulin's result that a symplectic automorphism $f: X \stackrel{\sim}{\longrightarrow} X$ of a K3 surface $X$ has $\operatorname{order} \operatorname{ord}(f) \leq 8$, see [1]. Voisin's recent result in [13] settles the case ord $(f)=2$ and, according to Corollary 1.5 , only the cases ord $(f)=4,6,8$ remain to be proven.

The case $\operatorname{ord}(f)=6$ follows immediately, as $\mathbb{Z} / 6 \mathbb{Z} \cong \mathbb{Z} / 3 \mathbb{Z} \times \mathbb{Z} / 2 \mathbb{Z}$ and Corollary 1.5 applies to the generators of the two factors. For ord $(f)=4,8$ one applies [13] repeatedly. Let us demonstrate this for $\operatorname{ord}(f)=4$, the case $\operatorname{ord}(f)=8$ is similar. Consider the quotient $X \longrightarrow \bar{X}:=X /\left\langle f^{2}\right\rangle$. Since $f^{2}$ is a symplectic involution, one knows by [13] that $[x]=\left[f^{2}(x)\right] \in \mathrm{CH}^{2}(X)$. Now apply [13] again to $\bar{X}$, which is a (singular) K3 surface, and the symplectic automorphism $\bar{f}: \bar{X} \stackrel{\sim}{\longrightarrow} \bar{X}$, which is of order 2 . Hence, the images $\bar{x}, \bar{f}(\bar{x}) \in \bar{X}$ are rationally equivalent. Pulled-back to $X$, this shows that $[x]+\left[f^{2}(x)\right]=[f(x)]+\left[f^{3}(x)\right]$ and hence $2[x]=2[f(x)]$, but $\mathrm{CH}^{2}(X)$ is torsion free. (Since it is enough to prove $[x]=[f(x)]$ for generic $x \in X$, the singularities of $\bar{X}$ are of little importance. Just apply the arguments to its minimal desingularization.)

In fact, as A. Sarti has pointed out to the author, the case ord $(f)=8$ could also be deduced from Theorem 1.4. Indeed, in this case $\Omega_{G}$ is of rank 18 (see [6, Prop. 5.1]) and hence $\operatorname{rk}(\mathrm{NS}(X)) \geq 19$. By Corollary 2.6, i) in [2] one knows that $T(X) \subset U^{\oplus 3}$ and by Theorem 6.3 of the same paper this implies $E_{8}(-1)^{\oplus 2} \subset \mathrm{NS}(X)$, which allows one to apply Theorem 1.4. In fact, the same reasoning also applies to $\operatorname{ord}(f)=7$.

\section{Acknowledgments}

Thanks to Alice Garbagnati, Bert van Geemen, and Claire Voisin for comments on a first version of this note. I am particularly grateful to Alessandra Sarti for comments on the case of order 4 and 8 .

\section{References}

[1] V.V. Nikulin, Finite groups of automorphisms of Kählerian K3 surfaces, Trudy Moskov. Mat. Obshch. 38 (1979), 75-137.

[2] D. Morrison, On K3 surfaces with large Picard number, Invent. Math. 75(1) (1984), $105-121$.

[3] C. Voisin, Sur les zéro-cycles de certaines hypersurfaces munies d'un automorphisme, Ann. Scuola Norm. Sup. Pisa Cl. Sci. 19(4) (1992), 473-492.

[4] B. van Geemen and A. Sarti, Nikulin involutions on K3 surfaces, Math. Z. 255(4) (2007), $731-753$.

[5] A. Garbagnati and A. Sarti, Symplectic automorphisms of prime order on K3 surfaces, J. Algebra 318(1) (2007), 323-350.

[6] - Elliptic fibrations and symplectic automorphisms on K3 surfaces, Comm. Algebra 37(10) (2009), 3601-3631.

[7] D. Huybrechts, Chow groups of K3 surfaces and spherical objects, J. Eur. Math. Soc. 12(6) (2010), 1533-1551.

[8] V. Guletskii, and A. Tikhomirov, Algebraic cycles on quadric sections of cubics in $\mathbb{P}^{4}$ under the action of symplectomorphisms, (2011), arXiv:1109.5725v1.

[9] D. Huybrechts, Chow groups and derived categories of K3 surfaces, in Current developments in algebraic geometry, Vol. 59 of Math. Sci. Res. Inst. Publ. 177-195, Cambridge University Press, Cambridge (2012). 
[10] C. Voisin, The generalized Hodge and Bloch conjectures are equivalent for general complete intersections, (2012), arXiv:1107.2600.

[11] C. Pedrini, On the finite dimensionality of a K3 surface, Manuscripta Math. 138(1-2) (2012), $59-72$.

[12] D. Huybrechts and M. Kemeny, Stable maps and Chow groups, (2012), arXiv:1202.4968.

[13] C. Voisin, Symplectic involutions of K3 surfaces act trivially on $\mathrm{CH}_{0},(2012)$, arXiv:1204.6684.

Mathematisches Institut, Universität Bonn, Endenicher Allee 60, 53115 Bonn, Germany E-mail address: huybrech@math.uni-bonn.de 
